\title{
The prediction of electric energy consumption using an artificial neural network
}

\author{
I. Chernykh ${ }^{1}$, D. Chechushkov ${ }^{1} \&$ T. Panikovskaya ${ }^{2}$ \\ ${ }^{1}$ Department of High Voltage Engineering, \\ Ural Federal University, Russia \\ ${ }^{2}$ Department of Automated Electric Systems, \\ Ural Federal University, Russia
}

\begin{abstract}
This paper presents the results of the studies on forecasting the electrical loads for a megapolis district with the use of artificial neural networks (ANN) as one of the most accomplished and promising solutions to this challenge. A theoretical approach to the issue is combined with the results of experimental studies using real schedules.

Keywords: artificial neural network, electrical loads, updated input data.
\end{abstract}

\section{Introduction}

The transition to the wholesale electricity market [1] has exacerbated the problems associated with the accuracy of forecasting electricity demand by tightening the requirements for compiling speed and the reliability of forecasts. New conditions associated with the introduction of heavy penalties require the introduction of modern electricity consumers of software and hardware for the collection of information on power consumption, for making accurate forecasts using flexible models and for reacting appropriately to changing trends.

The relevance of research in this area is shown in the application of the results to the operational and tactical management of power consumption that can reduce production costs and, as a consequence, increase the company's competitiveness by reducing production costs. Thus for the entity acquiring electricity on the wholesale and/or retail market, there is the task of compiling reliable applications for electricity consumption for a certain period ahead. The relevance of this research is supported by the peculiarities of distributed 
generation sources in island mode configuration. Due to more accurate forecasting, consumption can build the most accurate charts for loads and a more economical operation of power maneuvering.

\section{The proposed approach}

\subsection{Statement of the problem}

Recently, widespread methods for forecasting energy consumption are based on nonlinear models. Most of these methods belong to the category of so-called artificial intelligence techniques. This is primarily the artificial neural network. Typically, such methods require significant computational resources. The rapid development of modern computer technology greatly simplifies the process of building and implementing such models. In addition, the prediction accuracy of such methods significantly exceeds the accuracy of other methods [2]. Thus, the problem reduces to obtaining prediction models depending on electricity consumption from external factors, and previous consumption values. Building a model of energy consumption is planned to receive based on a neural network. Also, the processes of human life are very complex and multifactorial, so forecasting based on regression analysis (as the most appropriate in this case), to identify and mathematically describe the existing dependencies between variables, is a very complex and time consuming task. For this purpose, in using neural networks we restrict ourselves to the identification of links, leaving the mathematical description of the dependency algorithm to neural network training.

When analyzing the current literature on this topic [2, 3] it can be concluded that current scientific methods for predicting energy consumption are not yet developed enough to warrant their regular use: there is no common technology or methodology which can always guarantee quality results. Therefore, a promising direction for research in this area has opened up. In this paper the area of the metropolis is the object of investigation.

\subsection{Acquisition and processing of data for the neural network}

The training dataset is an acquisition of observations, which are the values of input and output variables. However, we must take into account some of the nuances, which are discussed below.

Firstly, it makes sense to consider all the variables that can affect the outcomes - at later stages this set is reduced. As the number of variables increases, the required number of observations increases linearly so that even with a relatively small number of variables there may be a huge number of observations. This difficulty is known as the "curse of dimensionality".

In many real problems we have to deal with quite unreliable data. Some variables may be corrupted by noise or partially absent. Emissions - values that lie very far from the range of normal values of a variable - can distort the result of training. In such cases it is best to try to detect and remove these emissions by removing the appropriate observations - if you have enough data, or replacing emissions averages between adjacent observations or statistics. 
In this paper, we considered the load forecasting model applied to one of the neighborhoods of the city of Yekaterinburg. To determine the significant factors in the calculation interviews were conducted with experts of the energy retail company "Sverdlovenergosbyt". To identify the importance of factors a correlation analysis of consumption depending on various factors of electricity consumption was performed.

Let us now consider each of these factors in more detail.

The effect of temperature due to the fact that human activity requires special conditions, one of which is to maintain the room temperature at a certain level: the influence of this factor on the periods before and after the start/end of the heating season can clearly be seen.

Character energy, divided into five types: Monday (Monday morning is separate due to it being the first working day after the holidays). Tuesday-Thursday have approximately the same consumption patterns, with peaks in the morning and evening hours. Friday's pattern of consumption differs from the average day of the week, due to the shortened day at many enterprises of the city, and in the warmer months most people go out of town in the evening. Saturday and Sunday are days off and have their own consumption patterns; it is noted that the difference between them is mainly due to the specifics of human behavior.

Note that days during the holidays are different from the nature of the power allocated to the above five types and should be a separate group.

The effect of natural light (daylight hours) on energy consumption is most pronounced in the morning and evening hours.

It is worth noting the effect of wind on power consumption, especially at the northern wind speeds of over $6 \mathrm{~m} / \mathrm{s}$, where consumption increases significantly.

Experts from the electricity supply company identify the following most affecting factors: the presence of precipitation and cloudiness. Clouds have a significant impact on the natural light. Rainfall also leads to an increase in consumption, with an amount greater than $1 \mathrm{~mm}$.

Regarding the periodic factors, we must include the status of the heating season, such as heating and cooling off on the second day when cooling consumption begins to rise sharply. We must also take into account the schedule of maintenance works on the central heating pipes because during the maintenance work the hot water supply is disconnected and there is an increase in electricity consumption in the afternoon and evening.

For the input variables of the neural network, the load forecasting model is used to solve and describe the change in the time of the actual load values, which in general seems to be a nonlinear function:

$$
P_{t}=f\left(P_{t-1}, T^{\text {target }}, L D_{t}, S D_{t}, P R_{t}, C_{t}, T D_{t}\right) \text {, }
$$

where $P_{t-1}$ - quantity of electricity consumption in the previous observation; $T^{\text {proj }}$ - target temperature; $L D_{t}$ - longitude of the day for the period of observation; $S D_{t}$ - status of the day for the period of observation; $P R_{t}-$ 
precipitation for the period of observation; $C_{t}$ - percent of clouded sky; $T D_{t}-$ time of day.

To analyze the correlation between members of a time series we build an autocorrelogram - a graph of the correlation ratio of the value of the delay (offset) of the series. For this purpose, the original time series is shifted by an amount of delay, while a new row is obtained between these two series and seeks correlation.

Obviously, we need to build an autocorrelogram to calculate the same number of values of the correlation ratio, as the shifts will reflect the autocorrelogram. Autocorrelogram was constructed in the software package application Processing Toolbox Matlab. Matlab in the workspace has established a set of values for electricity (4382 values) (Fig. 1).

Constructed in such a way, the autocorrelogram reflects the dependence of the correlation coefficient on the delay. The correlation coefficient has the same meaning as the empirical correlation ratio, but is used to assess the closeness of the connection described by the linear regression equation. This assumption is quite justified in finding the most important values of the delay, as in this case we are not interested in the exact values of the correlations and the values of delay in which their peaks appear.

The autocorrelogram obtains symmetry about the axis of ordinates and these points are just a part, which are located in the first quarter of the Cartesian coordinate system. It can be seen that the autocorrelation coefficient substantially decreases, linearly, with increasing delay. The only pronounced peak is observed near zero lag. Therefore, when training the neural network, these values of lag will be of the greatest interest.

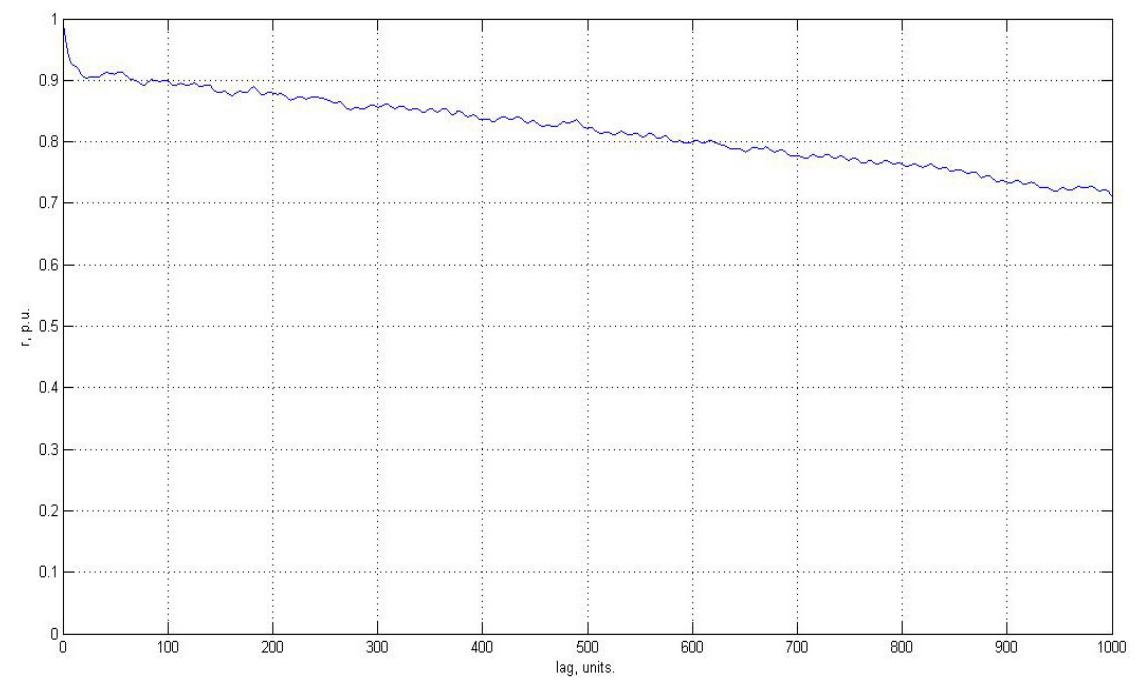

Figure 1: Autocorrelogram of electricity consumption, $r$ - autocorrelation coefficient; lag - lag value. 


\subsection{The structure of artificial neural network}

For the prediction of consumption, we used a classical multilayer artificial neural network-forward signal. The experiment was conducted with a number of network layers and neurons in the layer and these were examined using the following network architectures.

1. A network consisting of a single neuron (Fig. 2);

2. A network consisting of two layers: input and output (Fig. 3);

3. A network consisting of three layers: input, hidden and output (Fig. 4);

The number of neurons in the output layer network is strictly fixed; it is determined by the number of components of the output (target) vector. In our case, the predicted value is the power consumption of the neighborhoods within the metropolis. Therefore, the output layer network will always contain a single neuron.

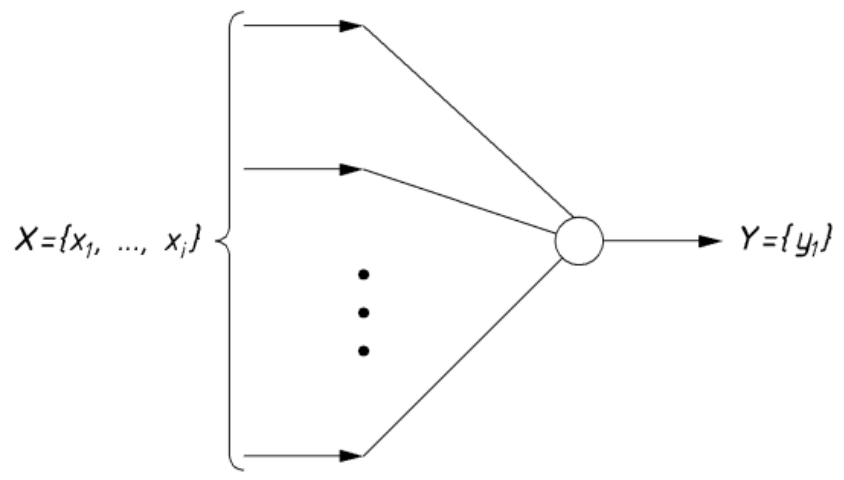

Figure 2: $\quad$ Network from one neuron; where $X$ is a vector of input data and $Y$ is a vector of output data.

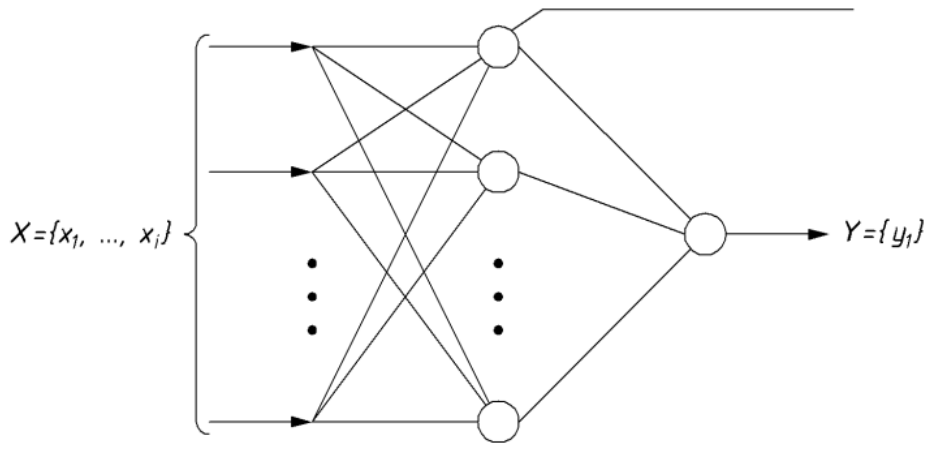

Figure 3: $\quad$ Two-layer network; where $X$ is a vector of input data and $Y$ is a vector of output data. 


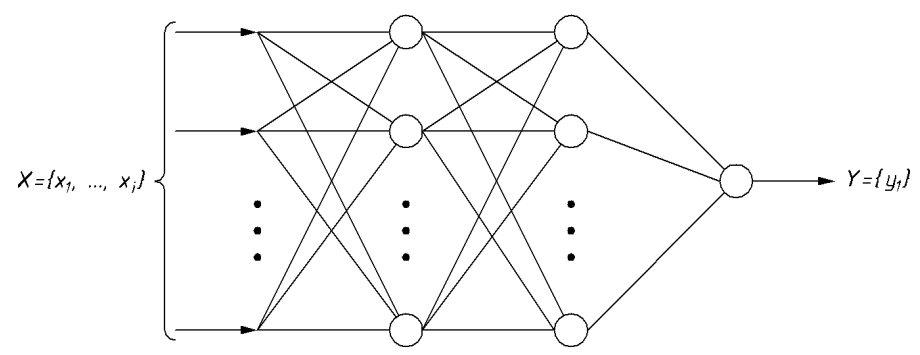

Figure 4: $\quad$ Three-layer network; where $X$ is a vector of input data and $Y$ is a vector of output data.

To determine the average relative prediction error on the test set for all 30 experiments, it should be noted that the training was conducted with the network again. This is due to the fact that an increasing number of experiments over the network significantly increases the time required for the training program network and the processing of results, with reduction - reduced accuracy in determining the expectation values and sample standard deviation $\sigma$.

Matlab was used for the construction and study of neural networks, and the program developed a block diagram as shown in Fig. 5.

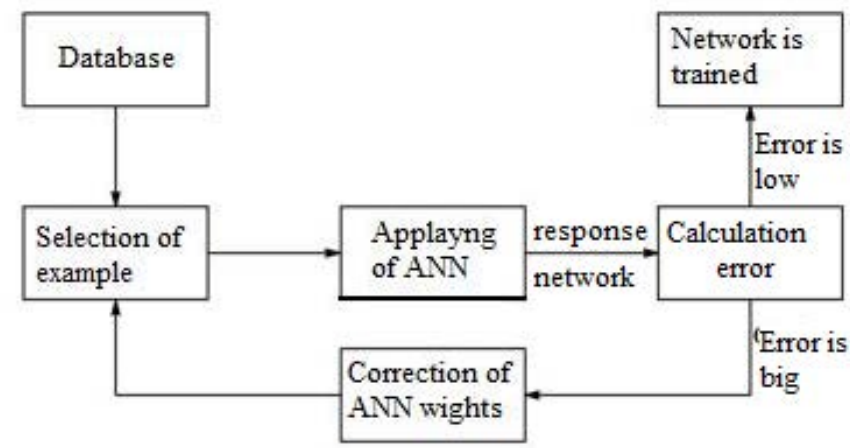

Figure 5: $\quad$ Block-diagram of the process of the learning network.

\section{Results and discussion}

\subsection{Influence of the number of neurons in the hidden layer of network}

Considering the impact of the number of neurons in the hidden layer network is only possible for three-layer networks. In constructing the networks involved in the experiments, we are guided by certain principles:

- The number of neurons in the input layer of the network is the number of input variables;

- The network has the forms of activation functions of neurons for each layer: purelin, logsig, purelin - for the input, hidden and output layers, respectively. 
The results of the experiments are shown in Fig 6. All three architectural embodiments (depending on the number of input variables) are featured in the network graph, which shows the acceptable values of the forecast.

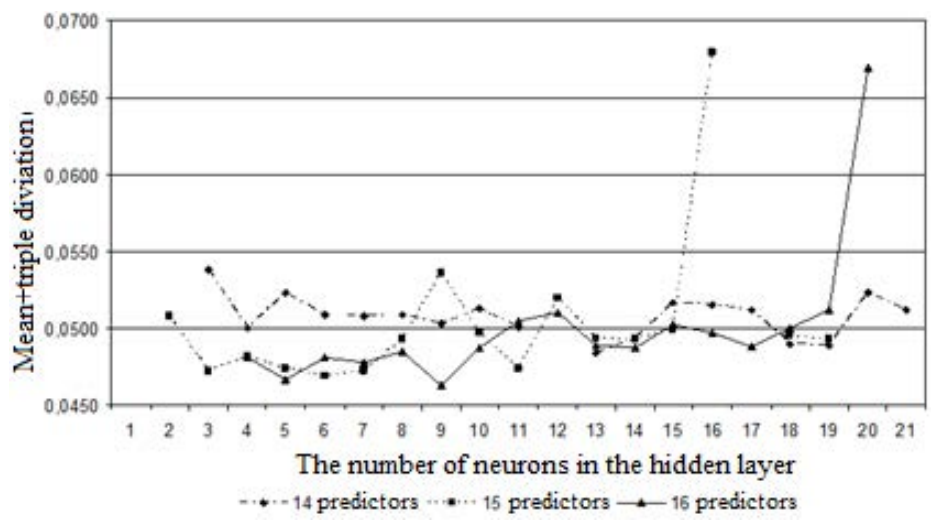

Figure 6: $\quad$ Summary graph of the effect of the number of neurons in the input layers of network by the amount forecast for three-layer networks.

When analyzing the receive data the following can be noted:

1. Changing the number of neurons in the hidden layer of the network has little effect on the value limiting relative prediction error on the test set and is not considered as unacceptable results. Figure 6 shows that the value fluctuates around an average value with a deviation of $0.5 \%$ (excluding two markedly remote values for networks with the number of predictors 15 and 16).

2. Not all variants of networks depending on the number of neurons in the intermediate layer are of acceptable accuracy for the forecast results issued. The network with one neuron in the hidden layer is not able to correctly compile data from the training set for new observations (high limiting relative prediction error on the test set).

3. Changing the number of predictor variables has little effect on the stability of networks in terms of the number of possible options for constructing them (changing the number of neurons in the intermediate layer) giving acceptable results. Meanwhile, the number of poor results forecasted does not change, they are simply redistribution.

As an example we will be plotting the actual values of the electric energy test set and the forecast given by the network. The neural network has the following architecture:

- number of input variables: 15 ;

- number of plies: 2;

- number of neurons in the input layer and activation function: 1 , with the logistic activation function;

- number of neurons in the output layer and the activation function:

1 , logistic activation function. 
The result is a schedule of the consumption of electrical energy from the city's neighborhoods, $W$, depending on the number of observations (Fig. 7), built according to the test set and the weekly range.

For long-term prediction the error average value was about $4 \%$ for daily forecasting and the mean error was about $3.5 \%$. This value is the error caused by the fact that the moment the neural network is used the predicted values of temperature, cloudiness, precipitation, wind strength and direction are affected. This in turn introduces an additional error. Practice shows that the plan and the weather can vary considerably.

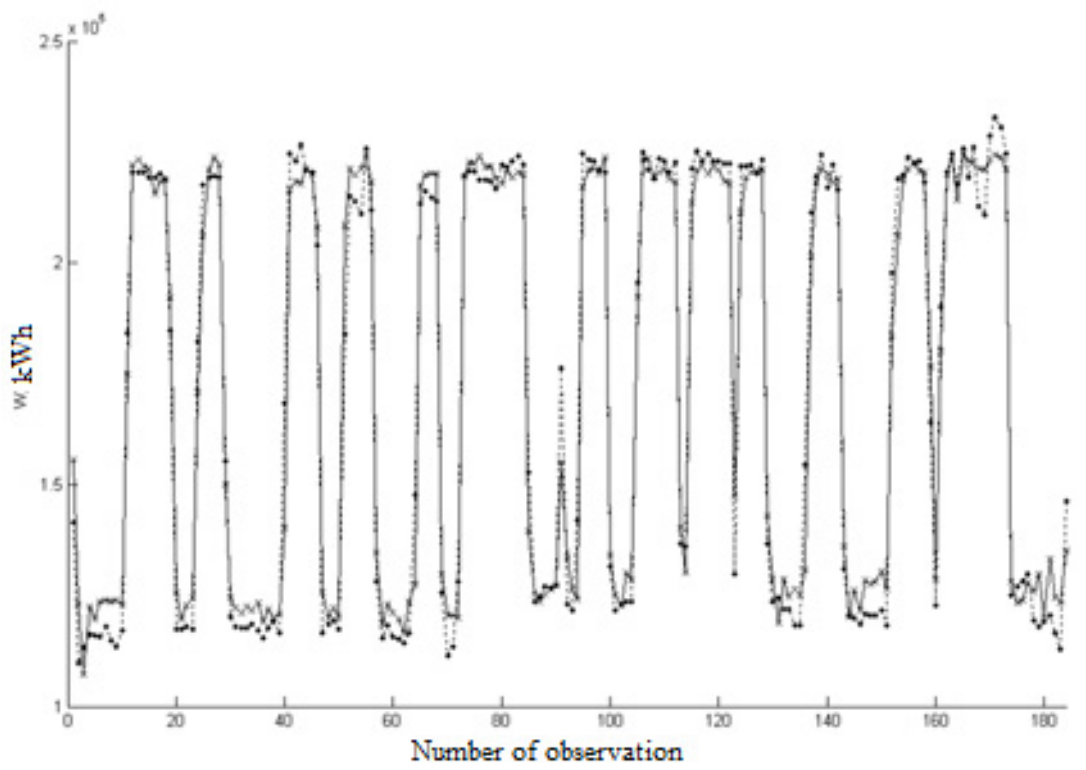

Figure 7: Actual and predicted electricity consumption. •- actual energy consumption, $\times-$ forecast electricity consumption.

\section{Conclusions}

Before immediately proceeding to the description of the conclusions and recommendations, it should be noted that a very important parameters has not yet been considered, which characterizes the operation of artificial neural networks - the time needed to train the network and present the user with the results of the forecast values. When placing the experiments on networks, this figure is not fixed, and the more complex the network architecture (increasing the number of layers and neurons, as well as input variables), the longer the time taken between the filing of the input network of the first sample of the training set and assigning output forecast values. Also, the amount of time affects the form of the activation functions of neurons: If you use a linear model then the learning network is much faster. 
General recommendations for the development of a system for forecasting electricity are as follows:

1. The importance of factors (variables) affecting the amount of the forecast electricity consumption and the need to include them in the training set can only be judged on the basis of experiments with networks.

2. The prediction results issued a classical multilayer neural network with direct signal propagation, learning algorithm back propagation and obey the normal distribution law. In this regard the best estimate of network performance in this case would be the expectation value and the standard deviation of the triple, that is $\bar{x} \pm 3 \sigma$.

3. A basic principle of network design: ceteris paribus, one should prefer a simpler model of two networks with approximately equal error control and it makes sense to choose one that is less - for the creation a system for forecasting part of an industrial facility, the two-layer network should be preferred.

4. Changing the number of neurons in the hidden layer of the network does not have a noticeable effect on the magnitude of the forecast.

5. Changing the number of neurons in the input layer network strongly influences the results issued by the network. We should pay attention to the two-layer network containing one neuron in the input layer.

One of the main observations in the work that is worth noting is that, as the number of input variables and the numbers of observations are increased, the accuracy of prediction also increases.

\section{References}

[1] Power market deregulation and its impact on the industrial enterprises functioning. Melnik Alexandr Nickolaevich Dr. Sci. Econ., Mustafina Olga Nikolaevna Scientific journal of Kuban State Technical University http://ej.kubagro.ru/2011/09/pdf/42.pdf

[2] Prediction of electrical loads in operational control of power systems based on neural network structures. Syktyvkar: KSC RAS, 2008

[3] K. Kavaklioglu, H. Ceylan, H.K. Ozturk, and O.E. Canyurt, "Modeling and prediction of Turkey's electricity consumption using Artificial Neural Networks,” Energy Conversion and Management, vol. 50, Nov. 2009, pp. 2719-2727 